\title{
Cianeto em tiquiras: riscos e metodologia analítica
}

Cyanide in "tiquira": risks and analytical methodology

\author{
Jomar Livramento Barros FURTADO ${ }^{1}$, Cícero Wellington Brito BEZERRA ${ }^{2}$, \\ Edmar Pereira MARQUES ${ }^{2}$, Aldalea Lopes Brandes MARQUES ${ }^{1 *}$
}

\begin{abstract}
Resumo
Tiquira é o destilado alcoólico obtido a partir da sacarificação e fermentação da mandioca, e além de íons cianeto, contém na sua composição as demais espécies potencialmente tóxicas presentes nas aguardentes, tais como: carbamatos, metanol e alguns íons de metais. Neste trabalho, uma metodologia analítica para a determinação de cianeto em amostras de tiquira é proposta, empregando voltametria no modo pulso diferencial, após destilação da amostra em presença de ácido nítrico $0,1 \mathrm{~mol} . \mathrm{L}^{-1}$. A metodologia desenvolvida foi comparada (teste $t$ ) com um método padrão para a determinação de cianeto. Os resultados indicam que os métodos são equivalentes do ponto de vista estatístico, confirmando a viabilidade da metodologia proposta. As condições estabelecidas (volume da solução absorvedora $=100 \mathrm{~mL} ; \mathrm{pH}=10$; faixa de potencial $=-0,5 \mathrm{a}-0,1 \mathrm{~V}$ e velocidade de varredura $=20 \mathrm{mV} \cdot \mathrm{s}^{-1}$ ) mostraram resultados reprodutíveis e permitiram a construção de curvas analíticas com valores de $\mathrm{R}$ da ordem de 0,999 . O valor médio de cianeto encontrado em tiquira foi $2,8 \times 10^{-5} \mathrm{~mol} . \mathrm{L}^{-1} \pm 2,4 \times 10^{-6} \mathrm{~mol} . \mathrm{L}^{-1}$, para $\mathrm{n}=5$, cerca de 150 vezes maior que o valor permitido para águas potáveis.
\end{abstract}

Palavras-chave: cianeto; aguardentes; voltametria.

\begin{abstract}
Tiquira is an alcoholic beverage made of fermented and distilled manioc root (Manihot esculenta). The presence of toxic species such as carbamates, methanol, heavy metals and cyanide in this beverage is considered crucial to the quality of this important product of northern Brazil. This article proposes an analytical procedure involving the use of voltammetry in the differential mode after the sample is distilled in the presence of $0.1 \mathrm{~mol} . \mathrm{L}^{-1}$ of nitric acid. The new methodology was compared (test $T$ ) with a standard method for determining cyanide, indicating that the methods are equivalent from thee standpoint of statistics and thus confirming the viability of the proposed methodology. The conditions established (volume of the adsorbent solution $=100.0 \mathrm{~mL} ; \mathrm{pH}=10$; potential range $=-0.5$ to $-0.1 \mathrm{~V}$; scan rate $=20 \mathrm{mV} \cdot \mathrm{s}^{-1}$ ) showed high reproducibility and allowed for the construction of analytical curves with values close to 0.999 . The "tiquira" showed an average cyanide content of $2.8 \times 10^{-5}$ mol.L $\mathrm{L}^{-1} \pm 2.4 \times 10^{-6} \mathrm{~mol} . \mathrm{L}^{-1}$ for $\mathrm{n}=5$, i.e., about 150 times higher than the acceptable content in drinking water.

Keywords: cyanide; firewater; voltammetry.
\end{abstract}

\section{Introdução}

A toxicidade do íon cianeto $\left(\mathrm{CN}^{-}\right)$é conhecida há mais de dois séculos. Este íon inibe a respiração celular atuando sobre as enzimas que contêm ferro (citocromo oxidase e catalase), impedindo que ocorra o consumo de oxigênio ${ }^{1,20}$. Por ingestão, a dose letal varia de 0,5 a 3,5 mg. $\mathrm{kg}^{-1}$ (cianeto/massa corpórea) ${ }^{9,20}$ e, por inalação, a concentração crítica desse íon está em torno de $10 \mu$ g. $L^{-1}$ de ar $^{1}$.

Existem diversas fontes contaminantes deste íon, dentre elas, efluentes das indústrias de poliacrilonitrilas, de síntese de resinas acrílicas, de nitrilas e aldeídos, de processamento de fármacos e de corantes, da extração de ouro e prata a partir dos seus minérios, da galvanoplastia, etc., e do processamento da mandioca (Manihot sculenta Crantz), euforbiácea originária da América do Sul, largamente cultivada no Brasil e considerada o vegetal que apresenta a maior concentração de glicosídeos cianogênicos $^{21}$.

A toxicidade cianogênica da mandioca origina-se da formação do ácido cianídrico (HCN) a partir da hidrólise enzimática de glicosídeos cianogênicos presentes, especialmente da

\footnotetext{
Recebido para publicação em 1/9/2006

Aceito para publicação em 5/9/2007 (001844)

${ }^{1}$ Departamento de Tecnologia Química - DETQI

Universidade Federal do Maranhão - UFMA

2 Departamento de Química - DEQUI,

Universidade Federal do Maranhão - UFMA, Maranhão - MA, Brasil,

E-mail: aldalea@quimica.ufma.br

*A quem a correspondência deve ser enviada
}

linamarina ${ }^{22,23}$. A hidrólise deste heterosídeo está apresentada na Figura 1 e, como pode ser visto, produz, além do ácido cianídrico, D-glicopiranose e propanona. A concentração de glicosídeos pode variar amplamente entre as cultivares, tanto por razões genéticas, como por fatores ambientais (localização, tipos de solo, estação), podendo atingir valores de até $2000 \mathrm{mg} \cdot \mathrm{kg}^{-1}$, tanto nos tubérculos, quanto nas folhas ${ }^{5,11}$.

A mandioca é empregada basicamente na fabricação de produtos alimentícios, além de ser utilizada para o consumo "in natura", tanto massas alimentícias quanto bebidas, e como recurso alternativo, também para obtenção de álcool combustível. Exemplos mais comuns de alimentos obtidos a partir deste vegetal são: farinhas d'água (produto fermentado) e seca (produto não fermentado), tucupi (suco dos turbéculos ralados e prensados, sem a fécula), tacacá (tucupi temperado e cozido), acubê, curandá, beijus e tapiocas. Como bebidas, destacam-se o tarubá, a caissuma ou cauim, cachiri paia e a tiquira, sendo esta última fermentada e destilada ${ }^{21}$.

No Estado do Maranhão, dentre os derivados da mandioca, destaca-se a tiquira, destilado com alto teor alcoólico (36 a 54\%) produzido de forma artesanal, e bastante apreciado regionalmente $\mathrm{C}^{7,23}$.

A presença de íons cianeto em tiquira representa dupla desvantagem, sendo a primeira sua natural toxicidade. $\mathrm{O} \mathrm{CN}^{-}$, complexado com metais (cobre e ferro, por exemplo), ainda que possa ter a sua toxicidade diminuída, nas condições de tampão fisiológico, quando ingerido, pode sofrer labilização 
e se coordenar ao ferro da hemoglobina, bloqueando assim o transporte de oxigênio no sangue. É o mesmo caso do nitroprussiato de sódio $\mathrm{Na}_{2}\left[\mathrm{Fe}(\mathrm{CN})_{5} \mathrm{NO}\right]$, droga amplamente usada por hipertensos e que apesar da sua reconhecida estabilidade devido à alta afinidade que os íons Fe(II,III) têm por este ligante ( $\approx \beta_{6}=10^{35}$ e $10^{43}$, respectivamente), em condições fisiológicas pode ocorrer a labilização de íons $\mathrm{CN}^{-}$, portanto, como prevenção, íons tiossulfato devem ser conjuntamente ministrados ao paciente para converterem $\mathrm{CN}^{-} \mathrm{em} \mathrm{SCN}^{-4,12}$.

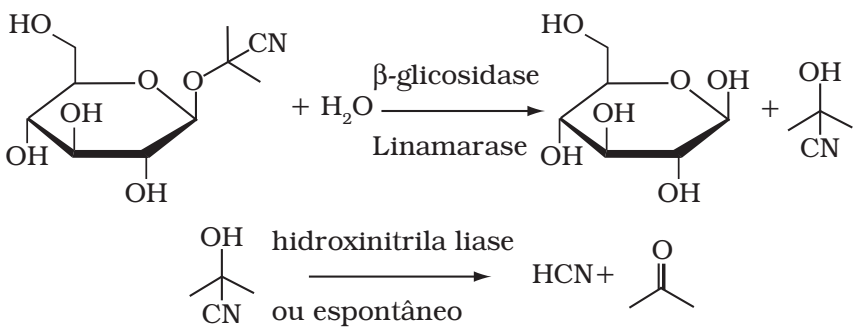

Figura 1. Esquema reacional para a degradação enzimática da linamarina.

O segundo aspecto, é que o íon $\mathrm{CN}^{-}$tem sido apontado como o mais importante precursor do carbamato de etila ou uretana $\left(\mathrm{EtOCONH}_{2}\right)^{2,6,18}$, composto que apresenta também elevada toxicidade, é cancerígeno e, recentemente, passou a ser controlado por legislação, com teor máximo permitido de 0,150 mg. $\mathrm{L}^{-1}$. O carbamato de etila é atualmente o principal responsável pela dificuldade de exportação de aguardentes brasileiros. A literatura ${ }^{2}$ registra um alto número de aguardentes com teores de carbamato de etila superior ao limite proposto. De 126 amostras de cachaças nacionais analisadas, por exemplo, apenas $21 \%$ atenderam a exigência para exportação com limite inferior a 0,150 mg. $\mathrm{L}^{-1}$. Não sem motivos, das 37 amostras de tiquiras analisadas, todas apresentaram teores de carbamato de etila muito superiores (de até setenta vezes) ao valor limite estabelecido. Estes fatos alertam para a necessidade urgente de se elucidar melhor a composição da tiquira e de se desenvolver metodologias analíticas para a identificação de espécies de interesse.

Devido à alta toxicidade do cianeto, os limites de concentração permitidos em ambientes de trabalho e em águas potáveis devem ser muito baixos ${ }^{14}$. Em bebidas alcoólicas não existe ainda um limite de referência permitido, mas para águas potáveis o limite de tolerância para cianeto é de $0,1 \mathrm{mg} \cdot \mathrm{L}^{-1}$ no Brasil ${ }^{8}$. Os baixos limites de cianeto exigidos demandam métodos de detecção e determinação cada vez mais sensíveis ${ }^{19}$. Por muitos anos tem havido um aumento na sensibilidade e variedades de métodos disponíveis para o monitoramento de cianeto, que pode aparecer não somente na forma de cianeto livre ( $\mathrm{HCN}, \mathrm{NaCN}, \mathrm{KCN}$ ) e de complexos instáveis como $\left[\mathrm{Zn}(\mathrm{CN})_{4}\right]^{-2}$, mas também como parte de complexos relativamente estáveis como os ferrocianetos e cobaltocianetos, os quais mesmo não apresentando as propriedades típicas do cianeto livre, são ainda tóxicos e classificados pela maioria das autoridades de saúde na mesma categoria deste ${ }^{19}$. É, portanto, reconhecida a necessidade de monitorar os teores de cianeto em alimentos e, certamente, na tiquira.
Desde a metade do século passado ${ }^{3}$, têm sido propostos e testados centenas de métodos analíticos para a determinação qualitativa e quantitativa de cianeto. Esses métodos envolvem, quase sempre, a destruição de complexos metálicos estáveis com liberação de cianeto de hidrogênio (HCN). KRUSE e MELLON ${ }^{16}$ propuseram a decomposição de complexos metálicos de cianeto por aquecimento com ácido fosfórico concentrado, sendo o cianeto de hidrogênio recuperado por destilação ${ }^{10}$.

O primeiro e principal problema na monitoração de cianetos é a escolha da metodologia analítica adequada ${ }^{3,20}$. Para esta finalidade, os métodos analíticos podem ser divididos em duas categorias principais: os métodos colorimétricos e os não colorimétricos ${ }^{3}$. Dentre os métodos não colorimétricos, podem ser citados os métodos voltamétricos e os polarográficos. Os métodos voltamétricos permitem a determinação de componentes presentes em uma solução, que podem ser oxidados ou reduzidos eletroquimicamente. Para isto, aplica-se um potencial à amostra, por meio de um eletrodo condutor, e o potencial elétrico que atua como força eletromotriz é varrido na região de interesse, permitindo obter assim um gráfico corrente versus potencial. Se em um determinado valor de potencial um componente da solução for oxidado ou reduzido, uma corrente será detectada no eletrodo de trabalho. O potencial onde isto acontece identifica o componente, e a quantidade de corrente produzida será proporcional à sua concentração na solução. No caso do cianeto, a base da sua determinação por voltametria é a oxidação do composto formado por reação com o mercúrio do eletrodo de trabalho, de acordo com a seguinte reação ${ }^{13}$ :

$\mathrm{Hg}+2 \mathrm{CN}^{-} \rightarrow \mathrm{Hg}(\mathrm{CN})_{2}+2 \mathrm{e}^{-}$

A técnica polarográfica é outro método clássico largamente empregado devido à facilidade de sua aplicação. Porém, a exemplo do que ocorre em outras determinações, a análise polarográfica de cianeto é sujeita a efeitos de matriz, principalmente quando aplicada ao controle de efluentes industriais ou análises clínicas ${ }^{15}$

No presente trabalho, foi utilizado um procedimento voltamétrico para a determinação de cianeto em tiquira. Os resultados foram comparados pelo método espectrofotométrico (método da piridina/ácido barbitúrico) que é considerado um método padrão para a determinação de cianeto.

Os demais métodos disponíveis, como por exemplo, o titulométrico e o enzimático, quando aplicados a uma mesma matriz, apresentam resultados discordantes, e até o momento não foi desenvolvida uma metodologia que seja rápida, simples, de baixo custo e acurada, que se aplique a diversas matrizes ${ }^{2}$.

\section{Material e métodos}

\subsection{Material}

Amostras de tiquiras foram coletadas diretamente dos produtores, no município de Santa Quitéria - MA. 
Para as coletas foram usados frascos de polietileno, previamente lavados durante vários dias com solução de ácido nítrico $\left(\mathrm{HNO}_{3}\right)$ 1:1 e extran neutro, sendo ainda ambientados na hora da coleta com a própria tiquira. As amostras foram estocadas sem nenhum pré-tratamento, à temperatura ambiente e observando as recomendações da literatura ${ }^{14,15}$.

\subsection{Métodos}

As medidas voltamétricas foram realizadas em um sistema analisador voltamétrico BAS, modelo CV-50W, composto dos seguintes acessórios: uma célula de vidro pirex, com capacidade de $10 \mathrm{~mL}$, contendo um eletrodo de gota pendente de mercúrio (eletrodo de trabalho), um fio de platina (eletrodo auxiliar) e um eletrodo de $\mathrm{Ag} / \mathrm{AgCl}$ imerso em solução saturada de $\mathrm{KCl}$ (eletrodo de referência).

As medidas espectrofotométricas foram realizadas em um espectrofotômetro modelo 8452-A Hewlett Packard. As medidas de $\mathrm{pH}$ foram realizadas em um peagômetro digital DIGIMED modelo BMPH-PV. Todos os reagentes empregados foram de pureza analítica e de procedência MERCK: ácido barbitúrico $\left(\mathrm{C}_{4} \mathrm{H}_{4} \mathrm{~N}_{2} \mathrm{O}_{3} \cdot 3 \mathrm{H}_{2} \mathrm{O}\right)$, ácido nítrico suprapur $\left(\mathrm{HNO}_{3}\right)$, ácido clorídrico $(\mathrm{HCl})$, ácido sulfâmico $\left(\mathrm{NH}_{3} \mathrm{SO}_{3}\right)$, piridina $\left(\mathrm{C}_{5} \mathrm{H}_{5} \mathrm{~N}\right)$ cloramina - $\mathrm{T}\left(\mathrm{C}_{7} \mathrm{H}_{7} \mathrm{ClNNaO}_{2} \mathrm{~S} \cdot 3 \mathrm{H}_{2} \mathrm{O}\right)$, cianeto de sódio $(\mathrm{NaCN})$, hidróxido de sódio $(\mathrm{NaOH})$, solução tampão borato $(\mathrm{pH}=10$ ). A solução estoque de cianeto foi preparada com concentração de $100 \mathrm{ppm}\left(2,0 \times 10^{-3} \mathrm{~mol} . \mathrm{L}^{-1}\right)$.

A água usada durante as análises foi ultrapurificada pelo sistema NANOPURE, modelo 04741 (BRANSTEAD).

\section{Tratamento da amostra para análise}

O íon cianeto foi determinado por voltametria após um procedimento de destilação, efetuado no destilador de Kjeldahl. Este destilador geralmente é usado para a destilação de amônia ou determinação de ácidos voláteis (acidez volátil) em amostras de alimentos. Para a destilação do cianeto, o destilador sofreu algumas adaptações para a remoção do cianeto da tiquira através da sua conversão em ácido cianídrico (HCN) o qual foi recolhido em uma solução de hidróxido de sódio (NaOH) 0,1 mol.L ${ }^{-1}$. Como esta destilação se dá por arraste de vapor, a solução absorvedora com volume inicial de $15 \mathrm{~mL}$ teve seu volume aumentado à medida que a destilação ia se processando, sofrendo assim, uma diluição cujos volumes finais avaliados foram de 50 a $250 \mathrm{~mL}$. A solução absorvedora foi então analisada por VPD. O procedimento mais adequado está descrito a seguir: $250 \mathrm{~mL}$ de água destilada foram adicionados em um balão gerador de vapor. Em seguida, $5 \mathrm{~mL}$ da amostra de tiquira foram transferidos para o tubo borbulhador e acidificados com $1 \mathrm{~mL}$ de ácido nítrico $\left(\mathrm{HNO}_{3}\right)$ e $1,000 \mathrm{~g} \mathrm{de}$ ácido sulfâmico $\left(\mathrm{NH}_{3} \mathrm{SO}_{3}\right)$. $\mathrm{O}$ sistema foi levado à ebulição. Por arraste de vapor, o ácido cianídrico que foi produzido após a acidificação da amostra foi recolhido em $15 \mathrm{~mL}$ de solução de hidróxido de sódio $(\mathrm{NaOH})$ 0,1 mol.L $\mathrm{L}^{-1}$. Esta solução é chamada de solução absorvedora, uma vez que absorve o gás cianídrico, neutralizando-o. Como esta destilação se dá por arraste de vapor, o volume final da solução absorvedora aumenta, atingindo no final da destilação $100 \mathrm{~mL}$, volume no qual a destilação é interrompida por ser suficiente para destilar todo o cianeto presente na amostra (como mostrou o teste com o padrão). A solução absorvedora foi então analisada por voltametria no modo pulso diferencial (VDP) para a determinação do teor de cianeto na tiquira.

\section{Determinação voltamétrica do cianeto}

Em uma célula eletroquímica foram adicionados $5,0 \mathrm{~mL}$ da amostra recém-destilada e $200 \mu \mathrm{L}$ de solução tampão borato e $\mathrm{pH}=10$. A amostra foi deaerada por 2 minutos com nitrogênio super seco para a remoção de oxigênio e, em seguida, em repouso por alguns segundos antes da etapa de varredura no sentido anódico.

A curva analítica foi construída por adição de padrões nas seguintes quantidades de cianeto: 10; 20 e $30 \mu \mathrm{L}$ de solução estoque de cianeto $\left(2,0 \times 10^{-3} \mathrm{~mol} . \mathrm{L}^{-1}\right)$. As concentrações finais, portanto, foram de $4,0 \times 10^{-6} ; 8,0 \times 10^{-6}$ e $1,2 \times 10^{-5}$ mol.L ${ }^{-1}$, respectivamente.

A Tabela 1 apresenta os parâmetros operacionais otimizados do equipamento para a determinação de cianeto na amostra de tiquira submetida a uma destilação.

\section{Ensaios de recuperação}

Estudos de recuperação foram realizados visando à confirmação da eficiência da metodologia proposta (destilação e análise voltamétrica). Tanto foram empregadas soluções padrão, quanto amostra real. Aquelas foram utilizadas com o objetivo de verificar se todo o cianeto presente no padrão era convenientemente destilado. As concentrações de $\mathrm{CN}^{-}$empregadas foram: $10^{-3} ; 10^{-4} ; 10^{-5} ; 10^{-6}$ e $10^{-7}$ mol.L-1 . Para verificar a influência da matriz no processo, os ensaios de recuperação foram realizados com a amostra real (tiquira). A metodologia empregada foi a seguinte: a $15 \mathrm{~mL}$ de tiquira foram adicionados $15 \mu \mathrm{L}$ de uma solução padrão de cianeto de concentração igual a $1 \times 10^{-2}$ mol. $\mathrm{L}^{-1}$. Esta amostra foi destilada e analisada por voltametria de acordo com o procedimento proposto. Este procedimento foi repetido por cinco vezes.

Tabela 1. Parâmetros experimentais para a determinação de cianeto em tiquira submetida à destilação do cianeto e analisada pela técnica voltametria no modo PD.

\begin{tabular}{lc}
\hline Parâmetros voltamétricos & Especificação do valor \\
\hline Potencial inicial & $-0,5 \mathrm{~V}$ \\
Potencial final & $-0,1 \mathrm{~V}$ \\
Sensibilidade & $10 \mu \mathrm{A} . \mathrm{V}^{-1}$ \\
Velocidade de varredura & $20 \mathrm{mV} \cdot \mathrm{s}^{-1}$ \\
Amplitude de pulso & $50 \mathrm{mV}$ \\
Direção da varredura & Anódica (positivo) \\
\hline
\end{tabular}

\section{Determinação espectrofotométrica do cianeto}

Com o objetivo de validar os resultados das determinações do íon $\mathrm{CN}^{-}$por voltametria no modo $\mathrm{PD}$, a espectrofotometria eletrônica na região do UV-VIS foi empregada. O método consiste em converter o $\mathrm{CN}^{-}$em $\mathrm{CNCl}$ pela reação com cloramina-T. Após a completa reação de formação do $\mathrm{CNCl}$, observa-se uma 
coloração vermelha-azulada mediante a adição do reagente piridina/ácido barbitúrico, cuja absorbância é determinada no comprimento de onda de $578 \mathrm{~nm}^{11}$.

\section{Resultados e discussão}

\subsection{Determinação de cianeto por voltametria no modo PD e ensaios de recuperação}

A determinação do cianeto em tiquira foi realizada após a destilação da amostra para liberação do ácido cianídrico, já que além de outras interações, o cianeto pode estar complexado com metais.

Primeiramente, foi realizado um estudo do volume final de solução absorvedora, a partir do qual a concentração de cianeto se manteria constante (Figura 2). O estudo voltamétrico foi realizado para os volumes finais de 50, 100, 150, 200 e $250 \mathrm{~mL}$, a partir do qual se obteve a Figura 2, que mostra os resultados correspondentes ao volume final de solução absorvedora versus concentração de cianeto.

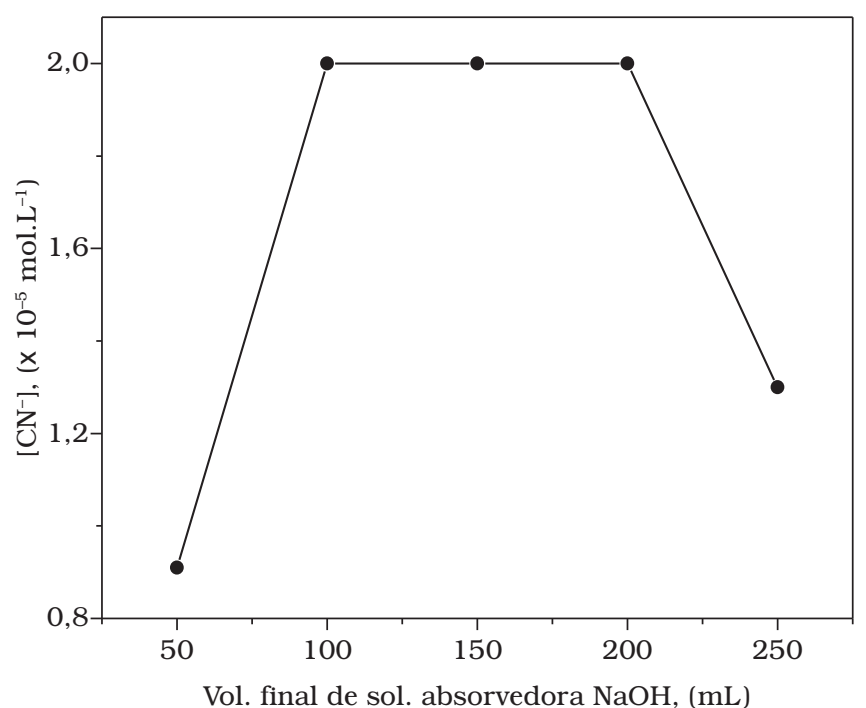

Figura 2. Estudo do volume final de solução absorvedora versus a concentração de cianeto. Técnica: voltametria no modo PD; volume de amostra: $5 \mathrm{~mL}$; sistema tampão: borato; $\mathrm{pH}=10 ; \mathrm{Ei}=-0,5 \mathrm{~V}$; $\mathrm{Ef}=-0,1 \mathrm{~V}$; e sens $=10 \mu \mathrm{A} \cdot \mathrm{V}^{-1}$.

Conforme pode ser observado na Figura 2, o volume final de solução absorvedora considerado ideal foi de $100 \mathrm{~mL}$, já que este valor corresponde ao início do patamar observado, o qual indica estabilidade da corrente. Os estudos de recuperação realizados em diferentes concentrações $\left(10^{-3} ; 10^{-4} ; 10^{-5}\right.$; $10^{-6}$ e $10^{-7}$ mol. $L^{-1}$ ) mostraram valores de concentração muito próximos aos valores originais dos padrões antes da destilação, indicando assim a eficiência da destilação. Os resultados estão apresentados na Tabela 2.

É importante ressaltar que em cada experimento de recuperação, para todas as concentrações padrão adicionadas de cianeto, foram avaliados os volumes finais de solução ab-
Tabela 2. Estudo da eficiência da destilação na determinação de cianeto por voltametria (estudo de recuperação).

\begin{tabular}{cc}
\hline$\left[\mathrm{CN}_{\mathrm{p}}^{-}\left(\mathrm{mol} . \mathrm{L}^{-1}\right)\right.$ & {$\left[\mathrm{CN}_{\mathrm{d}}\left(\mathrm{mol} . \mathrm{L}^{-1}\right)\right.$} \\
\hline $1,0 \times 10^{-3}$ & $9,7 \times 10^{-4}$ \\
$1,0 \times 10^{-4}$ & $9,7 \times 10^{-5}$ \\
$1,0 \times 10^{-5}$ & $9,9 \times 10^{-6}$ \\
$1,0 \times 10^{-6}$ & $9,6 \times 10^{-7}$ \\
$1,0 \times 10^{-7}$ & $9,8 \times 10^{-8}$ \\
\hline
\end{tabular}

$[\mathrm{CN}]_{\mathrm{p}}=$ Concentração original de cianeto da solução padrão; e $[\mathrm{CN}]_{\mathrm{d}}=$ Concentração determinada pela metodologia proposta.

sorvedora (50,100, 150, 200 e $250 \mathrm{~mL}$ ) os quais mostraram comportamentos idênticos ao observado na Figura 2. Por este motivo, o volume final de solução absorvedora escolhido foi $100 \mathrm{~mL}$, o que indica que todo o conteúdo de cianeto presente na amostra de tiquira tenha sido destilado no tempo correspondente a este volume.

Após a otimização do volume ideal de destilação (volume de solução absorvedora), foi realizado um estudo das condições de trabalho como o $\mathrm{pH}$ e o potencial inicial mais adequados, antes da determinação do cianeto por VPD. Para tanto foi avaliado um parâmetro de cada vez, visando obter as melhores resoluções de pico, bem como as maiores correntes como mostra a Figura 3.

No estudo do $\mathrm{pH}$ (Figura 3a) pode-se observar que a corrente de pico aumenta com o aumento do $\mathrm{pH}$ até o limite de $\mathrm{pH} 10$, decrescendo em seguida para maiores valores de $\mathrm{pH}$. Segundo a literatura ${ }^{13,17-19}$, na determinação de cianeto por voltametria, o valor de $\mathrm{pH}$ mais adequado está compreendido na faixa de 9 a 10, em concordância portanto com os resultados aqui obtidos. Assim, para a determinação de cianeto em tiquira, o valor de $\mathrm{pH}=10$ foi escolhido por fornecer voltamogramas bem definidos e as maiores correntes de pico. A fixação deste valor de $\mathrm{pH}$ foi realizada com o emprego de tampão borato $(\mathrm{pH}=10)$.

Após a otimização do $\mathrm{pH}$, foi avaliado o potencial inicial (de -0,8 a-0,5 V), deixando-se o potencial final fixo em -0,1 V. Os resultados estão apresentados na Figura 3b e indicam uma dependência da corrente de pico para o lado anódico, dentro do intervalo de potencial considerado. $\mathrm{O}$ valor definido foi $-0,5 \mathrm{~V}$, por garantir a maior corrente de pico.

Após a otimização das melhores condições de trabalho, procedeu-se a determinação do cianeto por VPD usando-se o eletrodo de gota pendente de mercúrio. As Figuras 4a e 4b mostram os voltamogramas e suas respectivas curvas de adição padrão, onde se observa boa resolução dos voltamogramas e uma excelente linearidade da curva de adição padrão 0,999.

A aplicação do procedimento otimizado em amostras de tiquira apresentou um valor médio de cianeto de $2,8 \times 10^{-5}$ mol.L $L^{-1} \pm 2,4 \times 10^{-6}$ mol. $L^{-1}$, para $\mathrm{n}=5$, um nível de confiança de $95 \%$ e um DPR (desvio padrão relativo) de 6,8\%.

Ensaios de recuperação envolvendo adição de padrão à amostra real confirmam também a eficiência do método. A concentração final de cianeto em uma amostra de tiquira igual a $3,8 \times 10^{-5} \mathrm{~mol} . \mathrm{L}^{-1}$ ( $1 \times 10^{-5} \mathrm{~mol} . \mathrm{L}^{-1}+2,8 \times 10^{-5} \mathrm{~mol} . \mathrm{L}^{-1}$ ), após destilada e analisada segundo a metodologia proposta, apre- 

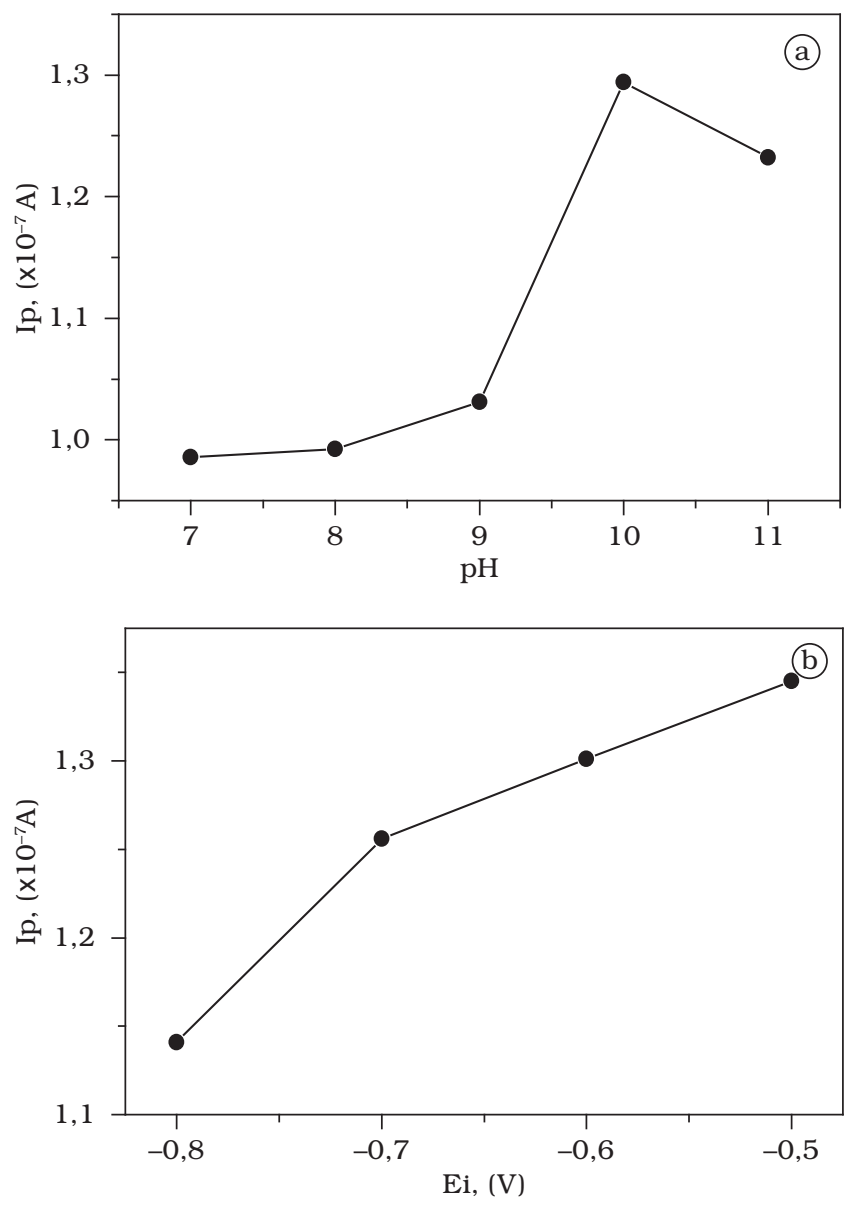

Figura 3. Estudo de efeito dos parâmetros experimentais sobre a corrente de pico na determinação de cianeto pela técnica voltamétrica no modo PD, em amostra de tiquira (destilada): a) $\mathrm{pH} \mathrm{e} \mathrm{b)} \mathrm{potencial}$ inicial; $\mathrm{V}_{\text {varred }}=20 \mathrm{mV} \cdot \mathrm{s}^{-1}$; sens. $=10 \mu \mathrm{A} \cdot \mathrm{V}^{-1} ;$ e $\mathrm{A}_{\text {pulso }}=50 \mathrm{mV}$.

sentou um teor de $3,5 \times 10^{-5} \mathrm{~mol} . \mathrm{L}^{-1}$, correspondendo a uma recuperação de $92 \%$.

Após uma avaliação preliminar, verificou-se que a amostra "in natura" ao ser acidificada com $\mathrm{HNO}_{3}$ pode levar este ácido à solução absorvedora de $\mathrm{NaOH}$. Neste caso, o $\mathrm{HCN}$ formado será somado ao excesso de $\mathrm{HNO}_{3}$, além dos demais ácidos voláteis (formados no processo fermentativo da tiquira), mesmo que em pequenas quantidades. Neste caso, haverá um maior consumo de $\mathrm{NaOH}$ para a neutralização de todos estes ácidos, tornando impossível serem distinguidos, através de uma titulação direta ou por retorno. Portanto, a escolha do método voltamétrico para determinação de cianeto foi considerada adequada porque somente ela pôde distinguir entre os dois ácidos diretamente envolvidos neste processo: $\mathrm{HNO}_{3}$ (adicionado) e HCN (formado).

\subsection{Determinaçáo de cianeto por espectrofotometria UV-VIS}

A concentração de cianeto encontrada por este método apresentou um valor médio de $2,9 \times 10^{-5} \mathrm{~mol} . \mathrm{L}^{-1} \pm 4,9 \times 10^{-6} \mathrm{~mol} . \mathrm{L}^{-1}$, para $\mathrm{n}=5$, um nível de confiança de $95 \%$ e um DPR de $13,8 \%$.
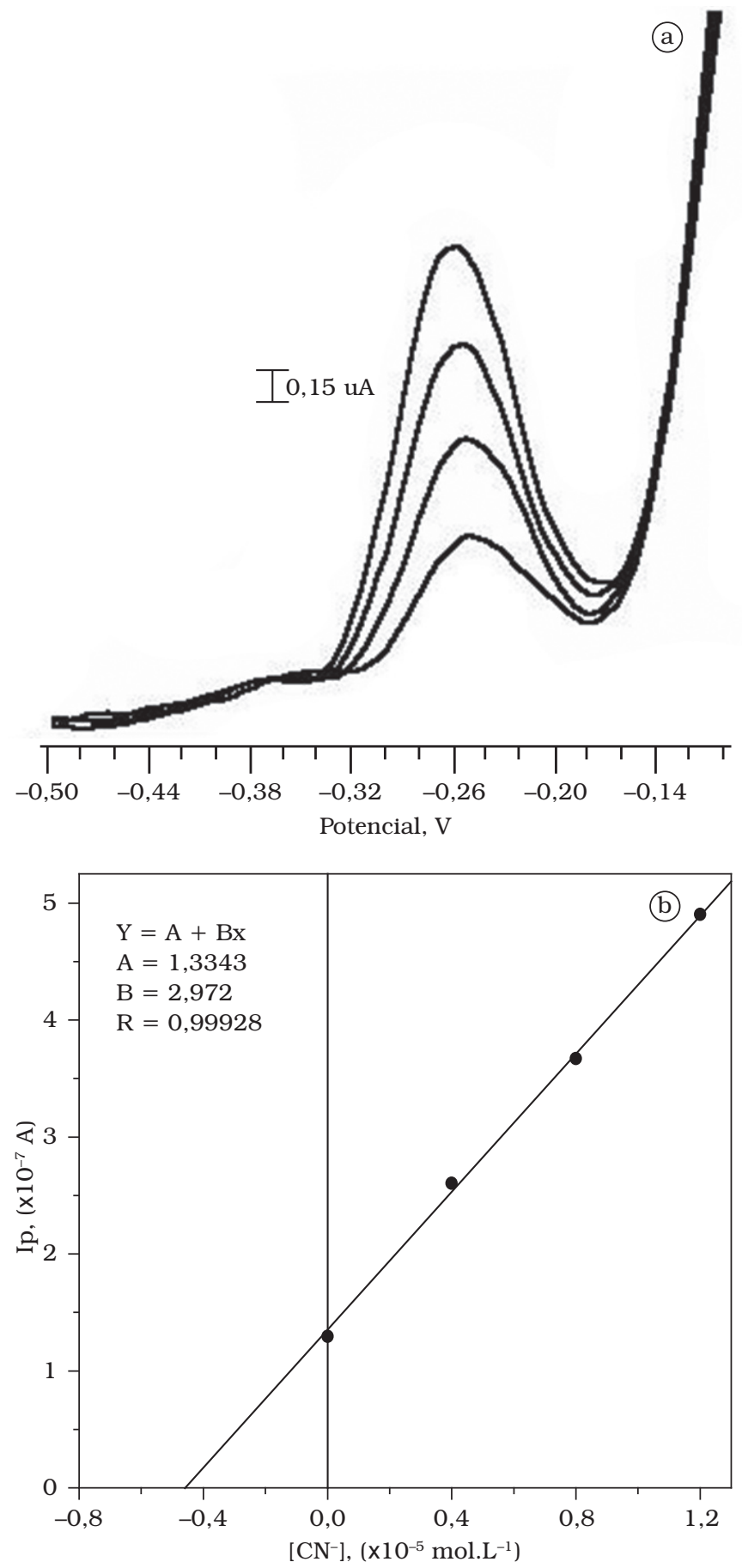

Figura 4. Resposta voltamétrica do cianeto em uma amostra de tiquira (destilada); a) voltamogramas por VPD. $\left[\mathrm{CN}^{-}\right]=4,0 \times 10^{-6} ; 8,0 \times 10^{-6}$ e $1,2 \times 10^{-5} \mathrm{~mol} . \mathrm{L}^{-1} ; \mathrm{Ei}=-0,5 \mathrm{~V}$; $\mathrm{Ef}=-0,1 \mathrm{~V} ; \mathrm{V}_{\text {varred }}=20 \mathrm{mV} \cdot \mathrm{s}^{-1} ; \mathrm{A}_{\text {pulso }}=50 \mathrm{mV}$; Sens $=10 \mu \mathrm{A} \mathrm{V}^{-1}$. $\mathrm{e}$ b) curva de adição padrão para a determinação do cianeto. Dados referentes à Figura 3a. Amostra diluída 6,66 vezes $\left(f_{d}=6,66\right)$.

A Tabela 3 mostra a comparação entre os resultados obtidos pelos dois métodos (voltamétrico e espectrofotométrico) na determinação de cianeto em tiquira.

Como a concentração de cianeto em bebidas alcoólicas brasileiras ainda não está regulamentada, o único parâmetro 
Tabela 3. Determinação de cianeto pelas técnicas de voltametria e espectrofotometria.

\begin{tabular}{ccc}
\hline \multirow{2}{*}{ Amostras } & \multicolumn{2}{c}{$\left[\mathrm{CN}^{-}\right] / 10^{-5}\left(\mathrm{~mol}^{-\mathrm{L}^{-1}}\right)$} \\
\cline { 2 - 3 } & Voltametria & Espectrofotometria \\
\hline A1 & 2,7 & 2,8 \\
$\mathrm{~A} 2$ & 3,0 & 3,2 \\
$\mathrm{~A} 3$ & 2,8 & 3,0 \\
$\mathrm{~A} 4$ & 2,7 & 2,8 \\
$\mathrm{~A} 5$ & 2,9 & 2,7 \\
$(\mathrm{X} \pm \mathrm{sd})$ & $2,8 \pm 0,16$ & $2,9 \pm 0,49$ \\
\hline
\end{tabular}

de comparação disponível é o limite máximo permitido de cianeto para águas doces - classe 1 , cujo valor é $1,9 \times 10^{-7}$ mol.L $\mathrm{L}^{-1}$ $\left(0,005 \mathrm{mg} \mathrm{CN}^{-} \mathrm{L}^{-1}\right)^{8}$, sendo portanto o teor encontrado neste destilado 150 vezes superior ao permitido para água potável, porém, mesmo assim uma comparação entre esses valores fica prejudicada considerando-se que uma pessoa ingere comparativamente pequenas quantidades de tiquira por dia.

\subsection{Avaliação estatística dos resultados}

A precisão dos resultados foi também avaliada através da reprodutibilidade das medidas voltamétricas. Nesse sentido, cinco alíquotas diferentes de tiquira foram analisadas quanto ao teor de cianeto pela técnica VPD. As medidas foram realizadas em cinco dias consecutivos. $\mathrm{O}$ valor do desvio padrão relativo (DPR) encontrado foi de $6,8 \%$, resultado considerado bastante satisfatório do ponto de vista analítico ${ }^{19}$. Os resultados estão apresentados na Tabela 3.

A exatidão foi avaliada comparando-se os resultados obtidos pelas duas metodologias empregadas (voltametria e espectrofotometria eletrônica), através da aplicação do teste $T$ de student. Para cinco determinações e para um nível de confiança de $95 \%(p=0,05)$, o valor crítico de $|t|$ é $2,78^{19}$. A média das diferenças para os pares de valores apresentados na Tabela 3 foi de $8,0 \times 10^{-7}$, e o desvio padrão das diferenças $1,6 \times 10^{-6}$. Estes números determinam um valor de 1,12 para $t$, com 4 graus de liberdade $(n=5)$. Como o valor calculado para $\mathrm{t}$ foi menor que o valor tabelado, isto significa, portanto, que não há diferença significativa entre as duas médias avaliadas, e que conseqüentemente os dois métodos são equivalentes do ponto de vista estatístico.

\section{Conclusões}

O cianeto não pôde ser determinado, diretamente, através da titulação da amostra de tiquira porque os ácidos presentes (nítrico adicionado, cianídrico produzido e os ácidos voláteis naturalmente presentes em aguardentes) não podem ser distinguidos por uma simples titulação. Portanto, a determinação voltamétrica foi considerada indispensável, sendo o método eletroquímico considerado uma ferramenta bastante viável. O procedimento otimizado proposto para a determinação de cianeto demonstrou ser vantajoso em relação ao procedimento de Kjeldahl, principalmente quanto à simplificação e à redução do tempo de destilação (apenas 20 minutos, contra 60 a 120 minutos exigidos pelo método tradicional). A avaliação estatística da precisão (reprodutibilidade) apresentou um valor de $6,8 \%$ de DPR, considerado satisfatório para o nível de concentração encontrado. A análise estatística da exatidão (comparação com a técnica UV-VIS segundo o teste $t$ de student) mostrou resultados satisfatórios, indicando não haver diferença significativa entre os resultados obtidos pelas duas técnicas. Pode-se concluir, com base nestes resultados, que os procedimentos voltamétricos aplicados no presente trabalho oferecem uma opção confiável para o controle de qualidade da tiquira e de outras matrizes semelhantes.

\section{Agradecimentos}

Os autores agradecem à CAPES pela bolsa de mestrado concedida.

\section{Referências bibliográficas}

1. A Toxidade do Íon Cianeto. Disponível em: $<$ http://www.mp3mix. com.br/trabalhos/quimica/ioncianeto.htm > . Acesso em: 4 abril. 2004.

2. ANDRADE SOBRINHO, L. G. et al. Carbamato de etila em bebidas alcoólicas (cachaça, tiquira e grapa). Quim. Nova, v. 25, n. 6b, p. 1074-1077, 2002.

3. BARK, L. S.; HIGSON, H. G. A Review of the Methods Available for the Detection and Determination of Small Amounts of Cyanide. Analyst, v. 88, p. 751-760, 1963.

4. BEZERRA, C. W. B. Manifestações da Ligação П em Aminas de Rutênio. São Carlos, 1999, 110 p. Tese (Doutor em Ciências, Área de Concentração Química Analítica), Instituto de Guímica de São Carlos (IQSC/USP).

5. BORGES, M. F.; FUKUDA, W. M. G.; ROSSETI, A. G. Avaliação de variedades de mandioca para consumo humano. Pesq. Agropec. Bras., v. 37, n. 11, p. 1559-1565, 2002.

6. BOSCOLO, M. Caramelo e carbamato de etila em aguardente de cana. Ocorrência e quantificação. São Carlos, 2001, 100 p. Tese (Doutor em Ciências, Área de Concentração Química Analítica), Instituto de Química de São Carlos, (IQSC/USP).

7. BRASIL. Decreto $\mathrm{n}^{\circ} 2314$ de 04 de setembro de 1997. Ministério da agricultura e abastecimento. Diário Oficial da República Federativa do Brasil, Brasília, DF, 05, set. 1997

8. BRASIL. Resolução n ${ }^{0} 357$, de 17 de março de 2005. Ministério do Meio Ambiente. Conselho Nacional do Meio Ambiente-CONAMA. Diário Oficial n ${ }^{\circ} 87$ da República Federativa do Brasil, Brasília, DF, 09, maio, 2005.

9. CASARINI, D. P.; DIAS, C. L. Estabelecimento de Padrões de Referência de Qualidade e Valores de intervenção Para Solos e Águas Subterrâneas no Estado de São Paulo. São Paulo, 1997. Relatório Parcial. CETESB.

10. CHARLOT. G. Chimie Analytique Quantitative. Masson et Cie, Paris, 1974, V. II, p. 375-379.

11. CONCISE INTERNATIONAL CHEMICAL ASSESMENTE DOCUMENT 61. Hydrogen cyanide and cyanides? Human health aspect. Geneva, 2004. Disponível em: <http://www. inchem.org/documents/cicads/cicads/cicad61.htm > . Acesso em: 12 dez. 2005.

12. FEelisCH, M.; STAMLER, J. S. Methods in Nitric Oxide Research. Grã-Bretanha, John Wiley \& Sons, 1996.

13. FRANK, M. H. T.; AFONSO, M. L. R.; OLIVEIRA, M. A. M. Método Voltamétrico para Determinação de Cianeto em Águas e 
Efluentes. In: $11^{\circ}$ Encontro Nacional de Química Analítica, EQ 31 - Campinas, 2001.

14. JACOBS, M. B.; The Analytical of Toxicology of Industrial Poisons. New York, Interscience Publishers, 1967, p. 721-741.

15. KOLTHOFF, I. M.; LINGANE, J. J.; Polarography. Interscience Plublishes. New York, 2nd edition, 1965, p. 541.

16. KRUSE. J. M.; MELLON M. G. Colorimetric Determination of Cyanide and Thiocyanate. Anal Chem., v. 25, p. 446-450, 1953.

17. LAFUEnTE, J. M. G. et al. Differential pulse voltammetric determination of low $\mu$ g 1-1 cyanide levels using EDTA, Cu (II) and hanging mercury drop electrode. Anal. Chim. Acta, v. 401, n. 1-2, p. 135-142, 2002.

18. MACKIENZIE, W. M.; CLYNE, A. H.; McDONALD, L. S. Ethyl carbamate formation in grain based spirits. Part II: The identification and determination of cyanide related species involved in ethil carbamate formation in Scotch Whisky. J. Inst. Brew., v. 96, p. 226-232, 1990.
19. MENDHAM, J. et al. Análise Química Quantitativa. VOGEL. $6^{\text {a }}$. ed. Londres: LTC, 2000.

20. PONCE, M. J. S. G. Determinação cinética de cianeto livre pelo monitoramento espectrofotométrico da reação de o-dinitrobenzeno com p-nitrobenzaldeído. Campinas, 2004, 109 p. Tese (Doutor em Ciências), Universidade Estadual de Campinas (Unicamp).

21. PRANCE, G. T. Manual de Botânica Econômica do Maranhão. São Luís, Gráfica Universitária UFMA/CORSUP, 1986.

22. SANTANA, M. A. et al. Linamarase expression in cassava cultivar with roots of low-and-high-cyanide content. Plant Physiol. v. 129, p. 1686-1694, 2002.

23. SANTOS, G. S. et al. Identificação e quantificação do cristal violeta em aguardentes de mandioca (tiquira). Quim. Nova, v. 28, n. 4 , p. 583-586, 2005.

24. WONG, W. C. D. Natural toxicants. In.: Mechanism and Theory in Food Chemistry. Nova Iorque, van Nostrand Reinhold, 1989. Cap. 8, p. 283-285. 